\title{
Ueber die Condensation von Aldehyden und Cyaniden;
}

von

\author{
C. Bechert.
}

(Mittheilung aus dem chem. Laboratorium von E, von Meyer.)

Die folgenden Versuche sind auf Anregung des Hrn. Prof. von Meyer und im Anschluss an die mannigfaltigen Untersuchungen über die Condensation von Aldehyden und Säuren, insbesondere an die in dieser Zeitschrift veröffentlichte Arbeit von Carrick über Cyanzimmtsäure ${ }^{1}$ ) ausgeführt worden.

I.

Einwirkung von Formaldehyd auf.Aethylencyanid:

$$
\text { Methylendisuccinimid: }\left(\mathrm{C}_{2} \mathrm{H}_{4}\left\langle\mathrm{CO}_{\mathrm{CO}}^{\mathrm{CO}}\right\rangle \mathrm{N}\right)_{2} \mathrm{CH}_{2} \text {. }
$$

Statt Formaldehyd wurde der polymere Paraformaldehyd angewandt, der unter den obwaltenden Bedingungen leicht, sobald die Reaction beginnt, in Formaldehyd zerfällt und so im nascirenden Zustande wirkt.

10 Grm. Aethylencyanid wurden zugleich mit 12 Grm. Paraformaldehyd in ca. 40 Grm. Eisessig gelöst, und zu der mässig warmen Flüssigkeit einige Tropfen concentrirte Schwefelsäure gefügt. Versetzt man die gekühlte Lösung mit kalter Schwefelsäure, so geht die Reaction ziemlich langsam von

1) Bd. 45, 500 .

Journal f. prakt. Chemie [2] Bd. 50. 
2 Bechert: Ueb. d. Condensat. von Aldehyden u. Cyaniden.

statten, und es dauert 2-3 Tage, ehe sich unter langsamem Entweichen von Formaldehyd Krystalle abscheiden. Giebt man jedoch zu der heissen Eisessiglösung die Schwefelsäure von etwa $15^{\circ}-20^{\circ}$, oder dieselbe gar heiss hinzu, so geht die Reaction in heftigster Weise vor sich, wobei unter starker Bräunung der Lösung der Formaldehyd auf einmal in Reaction tritt. Es empfiehlt sich daher, die Versuche bei Zimmertemperatur anzusetzen und nach einiger Zeit ein Mal gelinde zu erwärmen. Dadurch erreicht man, dass die Reaction in etwa zwei Stunden beendet ist, wobei sich grosse, wohl ausgebildete Krystalle aus der Lösung ausscheiden. Dieselben wurden auf dem Filter mit kaltem Wasser gewaschen, das einen Theil derselben löste; sowohl in diesem Waschwasser, als auch in der ursprünglichen Mutterlauge konnte Ammoniak, an Schwefelsäure gebunden, nachgewiesen werden. Der auf dem Filter zurückbleibende Körper, der sich in Alkohol und heissem Wasser leicht löst, wird aus letzterem umkrystallisirt und scheidet sich dabei in monoklinen Pyramiden, die zum Theil mit basischem Pinakoid versehen sind, aus. Er reagirt neutral, schmilzt bei $270^{\circ}$ noch nicht und ist, auf Platin erhitzt, scheinbar unzersetzt flüchtig.

$0,1900 \mathrm{Grm}$. Substanz gaben bei $11^{\circ}$ und $753 \mathrm{Mm}$. Druck $22,1 \mathrm{Ccm}$. $=13,76 \% \mathrm{~N}$.

$0,2466 \mathrm{Grm}$. Substanz gaben bei $13^{\circ}$ und $757 \mathrm{Mm}$. Druck $28,2 \mathrm{Ccm}$. $=13,46 \% \mathrm{~N}$.

0,1502 Grm. Substanz gaben 0,2832 Grm. $\mathrm{CO}_{2}=51,39 \% \mathrm{C}$ und 0,0682 Grm. $\mathrm{H}_{2} \mathrm{O}=5,06 \% \mathrm{H}$.

Die gefundenen Zahlenwerthe stimmen mit denen der Formel $\mathrm{C}_{9} \mathrm{H}_{10} \mathrm{~N}_{2} \mathrm{O}_{4}$ überein.

$\begin{array}{lcc} & \text { Berechnet: } & \text { Gefunden: } \\ \mathrm{C} & 51,43 & 51,39 \% \\ \mathrm{H} & 4,76 & 5,06 \% \\ \mathrm{~N} & 13,33 & 13,46 \%-13,76 \% .\end{array}$

Zur Ermittelung der Constitution des Körpers wurde derselbe mit verdünnter Schwefelsäure, 1 Theil conc. Schwefelsäure + 2 Theile Wasser, destillirt, wobei in der Vorlage Formaldehyd, in Wasser absorbirt, durch Geruch und seine reducirenden Wirkungen nachzuweisen war. Die im Destillationskolben zurückbleibende Flüssigkeit schied beim Erkalten Krystalle ab, die, aus heissem Wasser umkrystallisirt, bei $181^{\circ}$ 
Bechert: Ueb. d. Condensat. von Aldehyden u. Cyaniden. 3

schmelzen. Dieselben geben, mit Ammoniak versetzt und durch Eindampfen von überschüssigem Ammoniak befreit, mit Eisenchlorid die charakteristische Fällung von bernsteinsaurem Eisenoxyd. Die ausgeschiedenen Krystalle waren demzufolge Bernsteinsäure. Im Filtrat dieser Krystalle wurde Ammoniak, an Schwefelsäure gebunden, nachgewiesen.

Nach der Entstehung und Zersetzung des Körpers ist an der oben angenommenen Constitution kaum zu zweifeln. Seine Entstehung erklärt sich durch die Annahme, dass das aus Aethylencyanid durch Eintritt von Wasser und Ausscheidung von Ammoniak entstandene Succinimid sich mit Formaldehyd condensirt:

$$
2 \mathrm{C}_{2} \mathrm{H}_{4}\left\langle{ }_{\mathrm{CO}}^{\mathrm{CO}}>\mathrm{NH}+\mathrm{CH}_{2} \mathrm{O}=\mathrm{H}_{2} \mathrm{O}+\left(\mathrm{C}_{2} \mathrm{H}_{4}\left\langle{ }_{\mathrm{CO}}^{\mathrm{CO}}>\mathrm{N}\right)_{2} \mathrm{CH}_{2} .\right.\right.
$$

Das Methylendisuccinimid ist ein äusserst beständiger Körper: Chromsäure, sowohl in wässriger, wie in Eisessiglösung, vermag dasselbe nicht anzugreifen. Desgleichen wird es von rauchender Salpetersäure selbst beim Erhitzen nicht zerstört, und beim Einleiten von salpetriger Säure in seine Lösung, resp. Suspension in Eisessig, konnten nur Spuren von entstandener Bernsteinsäure nachgewiesen werden. Brom wirkt in der Kälte nicht auf dasselbe ein; erst im Rohr zngleich mit Wasser erhitzt, zerlegt es das Imid, wie nachgewiesen wurde, in Bernsteinsäure, Bromammonium und Kohlensäure.

Auch durch diese Zersetzung dürfte nachgewiesen sein, dass hier wirklich Methylendisuccinimid vorliegt.

\section{Einwirkung.von Benzaldehyd auf Aethylencyanid.}

Molekulare Mengen von Aethylencyanid (6 Grm.) und Benzaldehyd $(7 \mathrm{Grm}$.) wurden in 50-60 Ccm. absolutem Alkohol gelöst und dazu ca. 1,5 Grm. Natrium, in Form vnn Natriumäthylat in alkoholischer Lösung nach und nach hinzugefügt. Nach etwa zwei Tagen schied sich eine salbenartige Masse ab, und im Filtrat dieses Produktes schieden sich auf weiteren Zusatz von Benzaldehyd geringe Mengen eines graubraunen, feinen, hartkörnigen Niederschlages aus. Dieser wurde aus heissem Alkohol, in welchem er sich schwer löst, umkrystalli- 
4 Bechert: Ueb. d. Condensat. von Aldehyden u. Cyaniden.

sirt, wobei er sich in grauen, durchsichtigen Blättchen abschied, die bei $225^{\circ}$ unter Zersetzung schmolzen.

Da mehrere Ansätze verschiedene Resultate lieferten, indem sich manchmal nur geringe Mengen dieses salbigen Niederschlages bildeten, manchmal der oben erwähnte bei $225^{\circ}$ sich zersetzende Körper gar nicht entstand, ein drittes Mal sich zugleich faserig verflochtene, feine, gelbe Nädelchen bildeten, und da diese Versuche bei verschiedenen Temperaturen angesetzt worden waren, so musste durch besondere Versuche der Einfluss der Temperatur auf den Verlauf der Reaction ermittelt werden. Zu diesem Zwecke wurden molekulare Mengen von Aethylencyanid, Benzaldehyd und Natriumäthylat in alkoholischer Lösung einmal in Eis gekühlt zur Reaction gebracht, das andere Mal wurde das Natriumäthylat heiss zu der angewärmten alkoholischen Lösung gebracht. In beiden Fällen verlief die Reaction ganz verschieden von einander, und daher mögen sie auch getrennt von einander behandelt werden.

a) Condensation in der Kälte.

$$
\text { Verbindung: } \mathrm{C}_{21} \mathrm{H}_{22} \mathrm{~N}_{2} \mathrm{O}_{2} \text {. }
$$

Wie schon oben bemerkt, wurden molekulare Mengen von Aethylencyanid und Benzaldehyd in alkoholischer Lösung bei $0^{0}$ mit einem Molekül Natriumäthylat in alkoholischer Lösung von $0^{\circ}$ nach und nach versetzt. Aus der gelb bis roth gefärbten Lösung schieden sich nach zweitägigem Stehen geringe Mengen von blumenkohlartig angehäuften, schmutziggelben Krystalldrusen ab, die sich unter dem Mikroskop als ganz feine, filzartig verflochten€ Nädelchen erwiesen. Die Ausbeuten an Rohmaterial sind sehr gering; sie betrugen, selbst wenn die Mutterlauge noch Wochen lang stand, und Benzaldehyd noch hinzugefügt wurde, höchstens $15 \%$ der berechneten Ausbeuten. Der so gewonnene Körper ist in Aether und Eisessig ziemlich leicht löslich, in Alkohol schwerer, in Wasser unlöslich. Am besten lässt sich der Körper aus 70 procentigem Alkohol umkrystallisiren, wobei er sich in schönen, seideglänzenden, gelben, filzartig verflochtenen Nädelchen abscheidet. Er reagirt neutral und schmilat bei $214^{\circ}$. 
Bechert: Ueb. d. Condensat. von Aldehyden u. Cyaniden. 5

Der Körper ist sehr schwer verbrennbar; zur Erzielung guter Resultate mussten die Analysen im Bajonettrohr ausgeführt werden. $=8,24 \% \mathrm{~N}$.

0,1226 Grm. Substanz gaben bei $12^{\circ}$ und $761 \mathrm{Mm}$. Druck $8,5 \mathrm{Ccm}$.

$0,1040 \mathrm{Grm}$. Substanz gaben bei $13^{\circ}$ und $757 \mathrm{Mm}$. Druck 7,5 Cem. $=8,49 \% \mathrm{~N}$.

0,2261 Grm. Substanz gaben bei $12^{\circ}$ und $751 \mathrm{Mm}$. Druck $16 \mathrm{Ccm}$. $=8,30 \% \mathrm{~N}$.

0,1473 Grm. Substanz gaben 0,4084 Grm. $\mathrm{CO}_{2}=75,61 \% \mathrm{C}$ und 0,0898 Grm. $\mathrm{H}_{2} \mathrm{O}=6,77 \% \mathrm{H}$.

0,1762 Grm. Substanz gaben 0,4900 Grm. $\mathrm{CO}_{2}=75,75 \%$ C und 0,1087 Grm. $\mathrm{H}_{2} \mathrm{O}=6,85 \% \mathrm{H}$.

Aus diesen Zablenwerthen berechnet sich die Formel $\mathrm{C}_{21} \mathrm{H}_{22} \mathrm{~N}_{2} \mathrm{O}_{2}$.

\begin{tabular}{lcrrr} 
& Berechnet für $\mathrm{C}_{21} \mathrm{H}_{22} \mathrm{~N}_{2} \mathrm{O}_{2}:$ & \multicolumn{3}{c}{ Gefunden: } \\
$\mathrm{C}$ & 75,45 & - & 75,75 & $75,61 \%$ \\
$\mathrm{H}$ & 6,59 & - & 6,85 & $6,-7 \%$ \\
$\mathrm{~N}$ & 8,38 & 8,24 & 8,49 & $8,30 \%$
\end{tabular}

Beim Erhitzen des Körpers mit conc. Salzsäure im Rohr auf $150^{\circ}$ kounte nur Benzaldehyd und Ammoniak bestimmt nachgewiesen werden. Um einen weiteren Anhalt für die $\mathrm{Zu}$ sammensetzung dieser Verbindung zu erhalten, wurde versucht, in dem durch Salzsäure entstandenen Zersetzungsprodukte etwa entstandene Bernsteinsäure nachzuweisen; doch gelang es, trotz der sorgfältigsten Untersuchungen, nicht, auch nur eine Spur davon $\mathrm{zu}$ erhalten. Deshalb dürfte wohl anzunehmen sein, dass das Aethylencyanid gar nicht als solches in Reaction getreten ist, sondern nur das daraus resultirende Ammoniak. Die Verbindung $\mathrm{C}_{23} \mathrm{H}_{22} \mathrm{~N}_{2} \mathrm{O}_{2}$ erscheint daher als ein Condensationsprodukt von drei Molekülen Benzaldehyd mit zwei Molekülen Ammoniak, die sich unter Wasseraustritt vereinigt haben:

$$
3 \mathrm{C}_{6} \mathrm{H}_{5} \cdot \mathrm{CHO}+2 \mathrm{NH}_{3}-\mathrm{H}_{2} \mathrm{O}=\mathrm{C}_{21} \mathrm{H}_{22} \mathrm{~N}_{2} \mathrm{O}_{2} \text {. }
$$

Eine Bestimmung des Molekulargewichts dieses Körpers konnte den besten Aufschluss darüber geben, ob in der angegebenen Weise die beiden Ingredienzien sich vereinigt hätten. In Folge dessen wurden nach der Methode Raoult-Beckmann die Molekulargewichtsbestimmungen ausgeführt. Als Lösungsmittel konnte Eisessig nicht verwandt werden, da sich der Körper wohl darin löst, sich aber vor dem Gefrieren des 
6 Bechert: Ueb. d. Condensat. von Aldehyden u. Cyaniden.

Eisessigs wieder ausscheidet. Nur Nitrobenzol liess sich als Lösungsmittel gut anwenden. Die Lösungen wurden annähernd einprocentig hergestellt.

0,2324 Grm. Substanz gaben in $23,3508 \mathrm{Grm}$. Nitrobenzol eine Depression von $0,195^{\circ}$, wonach sich für $\mathbf{M}=361$ ergiebt. 0,1936 Grm. Substanz gaben in 19,4800 Grm. Nitrobenzol eine Depression von $0,205^{\circ}$, welcher $M=348$ entspricht. - Die obige Formel $\mathrm{C}_{21} \mathrm{H}_{22} \mathrm{~N}_{2} \mathrm{O}_{2}$ verlangt $\mathrm{M}=334$, während im Mittel gefunden wurde $\mathrm{M}=354$.

Um über die Constitution der Verbindung Aufschluss zu erhalten, wurde versucht, Derivate desselben darzustellen. Es wurde sowohl Acetylchlorid als auch Benzoylchlorld darauf einwirken gelassen; doch konnte ein positives Resultat nicht erzielt werden. Auch mit Acetanhydrid bildete der Körper nur schmierige Produkte, die nicht weiter verarbeitet werden konnten.

Vielleicht ist die obige Verbindung durch Condensation von einem Molekül Benzaldehyd mit zwei Molekülen des Additionsproduktes von Ammoniak und Benzaldehyd entstanden:

$\mathrm{C}_{6} \mathrm{H}_{5} . \mathrm{CHO}+2 \mathrm{C}_{6} \mathrm{H}_{5} . \mathrm{C}(\mathrm{OH}) \mathrm{H}\left(\mathrm{NH}_{2}\right)=\mathrm{H}_{2} \mathrm{O}+\mathrm{C}_{8} \mathrm{H}_{3} . \mathrm{CH}\left(\mathrm{C}_{6} \mathrm{H}_{5} . \mathrm{C}(\mathrm{OH}) \mathrm{NH}_{2}\right)_{2}$.

b) Condensation von Aethylencyanid und Benzaldehyd in der Wärme:

$\alpha$-Benzal- $\beta$-Cyanpropionsäureamid.

$\mathrm{CH}_{2} \cdot \mathrm{CN}$
$\left.\stackrel{\mathrm{C}}{\mathrm{CH}}, \mathrm{C}_{6} \mathrm{H}_{5}\right) \cdot \mathrm{CONH}_{2}$.

6 Grm. Aethylencyanid wurden mit 7 Grm. Benzaldehyd in absolutem Alkohol von $50^{\circ}$ gelöst, und zu der warmen Flüssigkeit 1,̃ Grm. Natrium als Natriumäthylat, unmittelbar nach der Lösung in Alkohol, auf ein $\mathrm{Mal}$ zugefügt, wobei unter starker Erwärmung und Bräunung der Lösung heftige Reaction eintrat. Nach einiger Zeit schied sich der Eingangs erwähnte salbige Niederschlag ab, der mit hartkörnigen, grauen Krystalldrusen durchsetzt war; die letzteren wurden von. ersterem durch Auftragen auf Thonplatten befreit. Unterm Mikroskop erwiesen sie sich als eine Anhäufung von schmutzig grauen, durchsichtigen, quadratischen Blättchen. Dieselben sind in Wasser, Aether, Petroläther und Benzol unlöslich, in 
Bechert: Ueb.d.Condensat. von Aldehyden u. Cyaniden. 7

heissem Alkohol äusserst schwer löslich und werden aus demselben nicht rein, sondern wieder als graues Krystallpulver erhalten. In heissem Eisessig dagegen sind sie leicht löslich und krystallisiren aus demselben in weissen, seideglänzenden Nädelchen aus, die sich in der Mutterlauge schneeballartig zusammenlagern. Der so rein erhaltene Körper verkohlt bei etwa $260^{\circ}$.

Zur Analyse wurde der getrocknete Körper sorgfältig von dem fest anhaftenden Eisessig befreit, indem er acht Tage im Exsiccator über Kalilauge stehen gelassen wurde. Diese Verbindung ist in noch höherem Maasse schwer verbrennbar als die vorher beschriebene, weshalb bei den langandauernden Verbrennungen im geschlossenen Rohr der Wasserstoff zu hoch gefunden wurde, während im offenen Rohr der Kohlenstoffgehalt um $1 \%-1,5 \%$ zu gering ausfiel, der Wasserstoffgehalt jedoch richtig ermittelt wurde.

$0,2416 \mathrm{Grm}$. Substanz gaben bei $17^{\circ}$ und $749 \mathrm{Mm}$. Druck 37,7 Ccm. = $15,0 \% \mathrm{~N}$.

$0,1709 \mathrm{Grm}$. Substanz gaben bei $17^{\circ}$ und $749 \mathrm{Mm}$. Druck 22,8 Ccm. $=$ $15,25 \% \mathrm{~N}$.

0,1134 Grm. Substanz gaben 0,2989 Grm. $\mathrm{CO}_{2}=71,88 \% \mathrm{C}$ und 0,0630 Grm. $\mathrm{H}_{2} \mathrm{O}=6,16 \% \mathrm{H}$.

0,1896 Grm. Substanz gaben 0,5002 Grm. $\mathbf{C O}_{2}=71,93 \% \mathbf{C}$ und 0,0988 Grm. $\mathrm{H}_{2} \mathrm{O}=5,75 \% \mathrm{H}$.

Im offenen Rohr gaben :

0,1800 Grm. Substanz 0,0908 Grm. $\mathrm{H}_{2} \mathrm{O}=5,61 \% \mathrm{H}$.

0,2074 Grm. Substanz 0,1028 Grm. $\mathrm{H}_{2} \mathrm{O}=5,5 \% \mathrm{H}$.

Die gefundenen Zahlenwerthe entsprechen am besten der Formel $\mathrm{C}_{11} \mathrm{H}_{10} \mathrm{~N}_{2} \mathrm{O}$.

\begin{tabular}{lcrccc} 
& Berechnet: & \multicolumn{5}{c}{ Gefunden: } \\
C & $\mathbf{7 1 , 6 1}$ & $\mathbf{7 1 , 8 8}$ & $\mathbf{7 1 , 9 3} \%$ & - & - \\
$\mathrm{H}$ & 5,38 & 6,16 & 6,75 & 5,61 & $5,5 \%$ \\
$\mathrm{~N}$ & 15,05 & 15,00 & $15,25 \%$ & -- & -
\end{tabular}

Die so empirisch ermittelte Formel kommt einem Condensationsprodukt von je einem Molekül Aethylencyanid und Benzaldehyd $\mathrm{zu}$, indem diese sich unter Wasseraustritt condensiren, dann aber die Elemente des Wassers wieder aufnehmen, gemäss folgender Gleichung:

$$
\underset{\mathrm{CH}_{2} \cdot \mathrm{CN}}{\mathrm{CH}_{2} \cdot \mathrm{CN}}+\mathrm{C}_{6} \mathrm{H}_{5} \cdot \mathrm{CHO}=\stackrel{\stackrel{\mathrm{CH}_{2}}{\mathrm{C}} \cdot \mathrm{CN}}{\mathrm{C}\left(\mathrm{CH} \cdot \mathrm{C}_{6} \mathrm{H}_{5}\right) \cdot \mathrm{CONH}_{2} .}
$$


8 Bechert: Ueb.d Condensat. von Aldehyden u. Cyaniden.

Dieser Körper sollte nach seiner Zusammensetzung im Gegensatz zu dem in der Kälte gewonnenen leicht verseifbar sein und dabei Bernsteinsäure liefern. Deshalb wurde er mit Kalilauge erwärmt, wobei er Ammoniak abgab. Ausserdem entstand ein Oel, das bald verharzte und intensiv nach Benzaldehyd roch. Salzsäure fällte aus der alkalischen Lösung einen weissen krystallinischen Niederschlag, der tus heissem Wasser umkrystallisirt wurde. Er schmolz bei $131^{\circ}$ und erwies sich dem Aussehen und allen Reactionen nac a als Bernsteinsäure.

Gegen verdünnte Säuren ist das obige Produkt beständiger, denn es löst sich in verdünnter Salzsäure und scheidet sich beim Zusatz von einigen Tropfen Ammoniak wieder in feinen Nadeln aus, welcher Umstand auch zum Reinigen von Rohmaterial benutzt werden kann.

Durch concentrirte Säuren, zumal durch Salpetersäure, wird er zersetzt, wobei Ammoniak und Bernsteinsäure nachgewiesen werden konnten. Auch durch salpetrige Säure, die in die Eisessiglösung eingeleitet wurde, um das vorliegende Amid in die zngehörige Säure überzuführen, wurde die Verbindung gänzlich zersetzt.

Molekulargewichtsbestimmung des Condensationsproduktes.

0,2000 Grm. Substanz gaben in 20,0588 Grm. Eisessig eine Depression von $0,17^{\circ}$, was $M=229$ ergiebt.

0,1653 Grm. Substanz gaben in $16,4224 \mathrm{Grm}$. Eisessig eine Depression von $0,17^{\circ}$, wonach $M=231$ berechnet wird.

Da das erwartete Molekulargewicht aber 186 ist, so kann man annehmen, dass das gefundene Molekulargewicht das der Verbindung +1 Mol. Lösungsmittel ist, welches alsdann zu 246 berechnet wird. Derartige Fälle kommen bekanntlich öfters vor.

Ein Derivat der Verbindung darzustellen, gelang hier ebenso wenig, wie bei der vorigen Verbindung.

Condensation von Anisaldehyd und Aethylencyanid: p-Methoxybenzalsuccinamid.

$$
\begin{aligned}
& \mathrm{C}_{6} \mathrm{H}_{4}\left(\mathrm{OCH}_{3}\right) \cdot \mathrm{CH}= \mathrm{C} . \mathrm{CONH}_{2} \\
& \mathrm{CH}_{2} \cdot \mathrm{CONH}_{2}
\end{aligned}
$$

Bei diesen Versuchen empfiehlt es sich, mit kleinen Quantitäten, etwa 3-5 Grm. zu arbeiten. Molekulare Mengen von 
Bechert: Ueb.d. Condensat. von Aldehyden u. Cyaniden. $\quad 9$

Aethylencyanid und Anisaldehyd wurden in Alkohol gelöst, und dazu nach und nach ein Molekül frisch bereitete Natriumäthylatlösung heiss zugefügt. Nach längerem Stehen setzt sich aus der dunkelbraun gefärbten Lösung ein gelbbrauner, salbiger Niederschlag $a b$, der nach und nach compacter wird, so dass er abgesaugt werden kann. Derselbe ist in Wasser und Aether nicht, in Alkohol und Eisessig leicht löslich und wurde aus letzterem umkrystallisirt, wobei er sich in weissen, seideglänzenden Nädelchen abschied. Der kaum basisch reagirende Körper schmilzt bei $255^{\circ}$.

$0,1352 \mathrm{Grm}$. Substanz gaben bei $18^{\circ}$ und $754 \mathrm{Mm}$. Druck 14,6 Ccm. = $12,37 \% \mathrm{~N}$.

0,2446 Grm. Substanz gaben bei $17^{\circ}$ und $754 \mathrm{Mm}$. Druck $25,8 \mathrm{Ccm} .=$ $12,14 \% . \mathrm{N}$.

$0,1190 \mathrm{Grm}$. Substanz gaben $0,2708 \mathrm{Grm} . \mathrm{CO}_{2}=62,01 \% \mathrm{C}$ und $0,0648 \mathrm{Grm} . \mathrm{H}_{2} \mathrm{O}=6,06 \% \mathrm{H}$.

0,2066 Grm. Substanz gaben $0,4674 \mathrm{Grm} . \mathrm{CO}_{2}=61,7 \%_{0} \mathrm{C}$ und 0,1156 Grm. $\mathrm{H}_{2} \mathrm{O}=6,21 \% \mathrm{H}$.

Die gefundenen Zahlenwerthe genügen der Formel: $\mathrm{C}_{12} \mathrm{H}_{14} \mathrm{~N}_{2} \mathrm{O}_{3}$.

\begin{tabular}{ccrc} 
& Berechnet: & \multicolumn{2}{c}{ Gefunden: } \\
$\mathrm{C}$ & 61,54 & $6 \mathbf{2 , 0 1}$ & $61,7 \%$ \\
$\mathrm{H}$ & 5,98 & 6,06 & $6,21 \%$ \\
$\mathrm{~N}$ & 11,96 & 12,37 & $\mathbf{1 2 , 1 4},$.
\end{tabular}

Man kann aus dieser empirisch gefundenen Formel schliessen, dass ein Condensationsprodukt von einem Molekül Aethylencyanid und einem Molekül Anisaldehyd vorliegt, die sich unter Wasseraustritt vereint haben, dafür aber zwei Mole küle Wasser aufgenommen haben.

$\underbrace{\mathrm{C}_{6} \mathrm{H}_{4} \cdot\left(\mathrm{O} \cdot \mathrm{CH}_{3}\right) \cdot \mathrm{CH}^{\mathrm{C}} \cdot \mathrm{CN}+2 \mathrm{H}_{2} \mathrm{O}=\mathrm{C}_{6} \mathrm{H}_{4}\left(\mathrm{O} \cdot \mathrm{CH}_{3}\right) \cdot \mathrm{CH}_{-}^{\mathrm{C}} \cdot \mathrm{CONH}_{2}}_{\mathrm{CH}_{2} \cdot \mathrm{CN}} \mathrm{CH}_{2} \cdot \mathrm{CONH}_{2}$.

primäres Produkt

Die manchen primären Amiden zukommende Eigenschaft, mit verdünnten Säuren Additionsprodukte zu bilden, kommt auch dem vorliegenden Körper zu. Mit verdünnter Salpetersäure mässig erwärmt, geht er in Lösung, und beim Erkalten derselben scheiden sich hellgelbe, feine Nädelchen ron $181^{\circ}$ Schmelzpunkt aus. Durch Wasser, zumal bei Zusatz von einigen Tropfen Ammoniak, wird daraus die ursprüngliche Verbindung zurückgebildet. 
10 Bechert: Ueb. d. Condensat. von Aldehyden u. Cyaniden.

$0,1633 \mathrm{Grm}$. Substanz gaben bei $15^{\circ}$ und $751 \mathrm{Mm}$. Druck $20,2 \mathrm{Ccm} .=$ $14,31 \% \mathrm{~N}$.

$\mathbf{N}$

$\begin{array}{cc}\text { Berechnet für } \mathrm{C}_{12} \mathrm{H}_{14} \mathrm{~N}_{2} \mathrm{O}_{3}, \mathrm{HNO}_{3}: \quad \text { Gefunden: } \\ 14,14 & 14,31 \% .\end{array}$

Mit verdünnter Salzsäure in gleicher Weise behandelt, bildet das Amid ein in gelben Nadeln krystallisirendes Salzsäureadditionsprodukt von $240^{\circ}$ Schmelzpunkt.

0,3932 Grm. Substanz gaben 0,2066 Grm. AgCl = 12,86\% Cl.

Cl

Berechnet für $\mathrm{C}_{12} \mathrm{H}_{14} \mathrm{~N}_{2} \mathrm{O}_{3}, \mathrm{HCl}$ : Gefunden:

Weitere Derivate darzustellen, gelang nicht; doch dürfte auf Grund der vorliegenden Zahlen an der oben angeführten Constitution kaum zu zweifeln sein.

Versuche, Aethylencyanid mit anderen Aldehyden, z. B. Zimmt- und Acetaldehyd, sowie mit Acetophenon zu condensiren, blieben erfolglos.

II.

Condensation von Aldehyden und Cyanessigester.

Zur Anwendung gelangten Anisaldehyd, Zimmtaldehyd, Furfurol, Piperonal und Salicylaldehyd; insbesondere mit letzterem wurden bemerkenswerthe Resultate erzielt. Man kann die dabei erhaltenen Condensationsprodukte, mit Ausnahme des mit Salicylaldehyd entstandenen, als Derivate der $c$-Cyanacrylsäure, resp. deren Aethylester betrachten, in welchen ein Wasserstoffatom der Methylengruppe durch die verschiedenen Aldehydradikale substituirt ist. ${ }^{1}$ )

Condensation von Anisaldehyd und Cyanessigester: p-Methoxyphenyl- $\alpha$-cyanacrylsäureester.

$$
\mathrm{C}_{6} \mathrm{H}_{4} \cdot\left(\mathrm{OCH}_{3}\right) \cdot \mathrm{CH}=\mathrm{C} \text {. (CN). } \mathrm{COOC}_{2} \mathrm{~B}_{5} \text {. }
$$

2 Grm. Cyanessigester und 2,5 Grm. Anisaldehyd wurden in ca. $20 \mathrm{Grm}$, absolutem Alkohol gelöst. Zu dieser Lösung

1) Es möge bemerkt werden, dass die Wahrnehmung Carrick's, wonach die besten Ausbeuten beim Arbeiten mit kleinen Quantitäten der Ingredienzien, etwa 5-5 Grm., erzielt werden, auch von mir bestätigt worden ist. 
Bechert: Ueb. d. Condensat. von Aldehydẹn u. Cyaniden. 11

wurden $0,01-0,02 \mathrm{Grm}$. Natrium als frisch bereitete Natriumäthylatlösung tropfenweise hinzugegeben. Nachdem die Lösung einen Tag gestanden hatte, waren aus derselben deutlich triklin ausgebildete Krystalle von lichtgelber Farbe ausgeschieden. Die Ausbeute war bei allen Ansätzen gut; sie betrug $80 \%-85 \%$ der berechneten. Der entstandene Körper löst sich in Alkohol, Aether, Chloroform und Benzol. Der Schmelzpunkt des neutral reagirenden Esters, der aus Alkohol umkrystallisirt wurde, liegt bei $85^{\circ}$. Durch Kochen mit Wasser wird er in seine Componenten zerlegt.

0,1784 Grm. Substanz gaben bei $18^{\circ}$ und $757 \mathrm{Mm}$. Druck 9,8 Cem. = $6,26 \% \mathrm{~N}$.

0,1580 Grm. Substanz gaben 0,3904 Grm. $\mathrm{CO}_{2}=67,38 \% \mathrm{C}$ und 0,0824 Grm. $\mathrm{H}_{2} \mathrm{O}=5,79 \% \mathrm{H}$.

$\begin{array}{ccc} & \text { Berechnet für } \mathrm{C}_{13} \mathrm{H}_{13} \mathrm{NO}_{3}: & \text { Gefunden: } \\ \mathrm{C} & 67,53 & 67,38 \% \\ \mathrm{H} & 5,63 & 5,79 \% \\ \mathrm{~N} & 6,06 & 6,26 \%\end{array}$

Hieraus ergiebt sich, dass das erwartete Condensationsprodukt, aus gleichen Molekülen Anisaldehyd und Cyanessigester entstanden, vorliegt.

$$
\begin{gathered}
\mathrm{C}_{6} \mathrm{H}_{4}\left(\mathrm{O} . \mathrm{CH}_{3}\right) \cdot \mathrm{CHO}+\mathrm{CH}_{2} \cdot(\mathrm{CN}) \cdot \mathrm{COOC}_{2} \mathrm{H}_{5}=\mathrm{H}_{2} \mathrm{O}+ \\
\mathrm{C}_{6} \mathrm{H}_{4}\left(\mathrm{O} \cdot \mathrm{CH}_{3}\right) \cdot \mathrm{CH}=\mathrm{C} \text {. (CN) } \cdot \mathrm{COOCO}_{2} \mathrm{H}_{5} .
\end{gathered}
$$

Darstellung der p-Methoxyphenyl- $\alpha$-Cyanacrylsäure:

$$
\mathrm{C}_{6} \mathrm{H}_{4}\left(\mathrm{O} \cdot \mathrm{CH}_{3}\right) \text {. } \mathrm{CH}=\mathrm{C} \text {. }(\mathrm{CN}) \text {. } \mathrm{COOH} \text {. }
$$

Um aus dem Ester die freie Säure zu erhalten, wurde zunächst das Kaliumsalz der letzteren durch Verseifen des ersteren dargestellt. Hierzu wurde derselbe in der genügenden Menge absoluten Alkohols gelöst, und in die heisse Lösung die auf ein Molekül Ester berechnete Menge alkoholischen Kalis heiss zugegeben. Es schied sich sofort ein weisser, flockiger Niederschlag aus, der beim Erkalten der Lösung zunahm. Dass nur das Carboxäthyl COO $\mathrm{C}_{2} \mathrm{H}_{5}$ verseift worden ist, die Cyangruppe also intact geblieben ist, zeigt schon die Zusammensetzung des Silbersalzes, welches aus der wässrigen Lösung des Kaliumsalzes durch salpetersaures Silber gefällt wurde.

0,4554 Grm. Silbersalz gaben 0,1600 Grm. Ag = 35,1\% Ag. 
12 Bechert: Ueb. d. Condensat. von Aldehyden u. Cyaniden.

$$
\begin{array}{ccc} 
& \text { Berechnet für } \mathrm{C}_{11} \mathrm{H}_{8} \mathrm{NO}_{3} \mathrm{Ag}: & \text { Gefunden: } \\
\mathrm{Ag} & \mathbf{3 4 , 8 4} & 35,1 \%
\end{array}
$$

Um aus dem Kaliumsalz die freie Säure zu erhalten, wurde dasselbe in Wasser von etwa $80^{\circ}$ gelöst, und zu dieser Lösung Essigsäure hinzugefügt. Die Säure schied sich darauf sofort als honiggelber, flockiger Niederschlag aus, welcher mit Wasser ausgewaschen und dann aus Alkohol umkrystallisirt wurde, wobei sich die reine Säure in schön ausgebildeten gelben Nadeln von $226^{\circ}$ Schmelzpunkt ausschied. Die Säure ist sehr schwer verbrennbar; daher mussten die Analysen im. Bajonettrohr vorgenommen werden.

$0,2826 \mathrm{Grm}$. Substanz gaben bei $16^{\circ}$ und $758 \mathrm{Mm}$. Druek $17,4 \mathrm{Ccm}$. = $7,14 \% \mathrm{~N}$.

0,3267 Grm. Substanz gaben 0,2128 Grm. $\mathrm{CO}_{2}=65,14 \% \mathrm{C}$, und 0,1390 Grm. $\mathrm{H}_{2} \mathrm{O}=4,72 \% \mathrm{H}$.

$\begin{array}{ccc} & \text { Berechnet für } \mathrm{C}_{11} \mathrm{H}_{9} \mathrm{NO}_{3}: & \text { Gefunden: } \\ \mathrm{C} & 65,02 & 65,14 \% \\ \mathrm{H} & 4,43 & 4,72 \% \\ \mathrm{~N} & 6,89 & 7,14, \ldots\end{array}$

Die vorliegende Säure ist im Gegensatz zum Ester in Chloroform schwer löslich. Rauchende Salpetersäure führt dieselbe in Nitranissäure über, was durch den Schmelzp. 186 ${ }^{\circ}$ und durch das Aussehen derselben nachgewiesen wurde.

Aus dem Kaliumsalz lässt sich leicht eine Reihe von Metallsalzen darstellen; so erhält man durch essigsaures Blei ein weisses Bleisalz, durch Chlorbaryum ein weisses Baryumsalz, durch Eisenchlorid ein orangefarbenes Eisensalz, durch schwefelsaures Kupfer ein lichtgrünes Kupfersalz; ferner wurden das Zinnsalz, das Quecksilber- und Kalksalz in Form weisser amorpher Fällungen gewonnen.

\section{Verbalten des p-Methoxyphenyl-c-cyanacrylsäure- esters gegen Brom.}

Da sich der von Carrick dargestellte $\alpha$-Cyanzimmtsäureester gegen Brom unerwarteter Weise ganz indifferent verhielt, so lag es natürlich nahe, auch den vorliegenden Ester auf sein Verhalten gegen Brom zu untersuchen. Zu diesem Zwecke wurde derselbe in der genügenden Menge kalten Chloroforms gelöst, und zwei Moleküle Brom, gleichfalls in wenig Chloro- 
Bechert: Ueb. d. Condensat. von Aldehyden u. Cyaniden. 13

form gelöst, hinzugefügt. In dem verschlossen gehaltenen Gefäss zeigte sich reichliche Bromwasserstoffentwicklung. Als das Gemisch etwa 12 Stunden lang gestanden hatte, schieden sich aus demselben weisse Nadeln aus. Dieselben wurden aus Alkohol umkrystallisirt und so als weisse, seideglänzende Nadeln von $185^{\circ}$ Schmelzpunkt erhalten.

$0,2258 \mathrm{Grm}$. Substanz enthielten 0,05902 Grm. $\mathrm{Br}=26,14 \% \mathrm{Br}$.

$0,2010 \mathrm{Grm}$. Substanz enthielten 0,0529 Grm. $\mathrm{Br}=26,01 \% \mathrm{Br}$.

$$
\begin{gathered}
\text { Br Berechnet für } \mathrm{C}_{12} \mathrm{H}_{12} \mathrm{NO}_{3} \mathrm{Br} \text { : } \\
25,81
\end{gathered} \quad \begin{gathered}
\text { Gefunden: } \\
\mathrm{Br}
\end{gathered}
$$

Jedenfalls bat sich zuerst das Additionsprodukt

$$
\mathrm{C}_{8} \mathrm{H}_{4} \cdot\left(\mathrm{OCH}_{3}\right) \cdot \mathrm{CHBr} \cdot \mathrm{CBr} \cdot(\mathrm{CN}) \cdot \mathrm{COOC}_{2} \mathrm{H}_{5}
$$

gebildet, welches dann durch Abspaltung von Bromwasserstoff in

übergegangen ist.

$$
\mathrm{C}_{6} \mathrm{H}_{4}\left(\mathrm{O}, \mathrm{CH}_{8}\right), \mathrm{CBr}=\mathrm{C},(\mathrm{CN}) . \mathrm{COOC}_{2} \mathrm{H}_{5}
$$

Im Gegensatz zu obigem Ester verhält sich die p-Methoxyphenyl- $\alpha$-cyanacrylsäure gegen Brom unter gleichen Bedingungen indifferent. Da dieselbe in Chloroform fast unlöslich ist, so wurde absoluter Alkohol bis zur Lösung zugefügt, und dann Brom auf sie einwirken lassen. Obgleich das Gemisch lange Zeit stehen gelassen wurde, konnte doch nur die ursprüngliche Säure aus der Lösung durch Eindampfen wiedergewonnen werden.

Condensation von Zimmtaldehyd und Cyanessigester:

Cinnamenyl-a-cyanacrylsäureester:

$$
\mathrm{C}_{8} \mathrm{H}_{5} . \mathrm{CH}=\mathrm{CH} . \mathrm{CH}=\mathrm{C} .(\mathrm{CN}) . \mathrm{COOC}_{2} \mathrm{H}_{5} \text {. }
$$

Molekulare Mengen von Zimmtaldehyd (6 Grm.) und Cyanessigester (5 Grm.) wurden in ca. $25 \mathrm{Grm}$. absolutem Alkohol gelöst, und hierzu, wie im vorigen Falle, ganz geringe Gewichtsmengen Natrium als Aetbylat in alkoholischer Lösung zugefügt. An der Einfallsstelle trat Trübung und geringe Bräunung ein, die beim Schütteln wieder verschwand. Sobald die Trübung eine bleibende war, wurde der Zusatz von Natriumäthylatlösung unterbrochen. Nach kurzer Zeit schieden sich in der Lösung gelbe Nadeln aus, die in Alkohol, Aether, Chloroform und Benzol löslich sind. Aus absolutem Alkohol 
14 Bechert: Ueb. d. Condensat. von Aldehyden u.Cyaniden.

umkrystallisirt, schmilzt das neutral reagirende Condensationsprodukt bei $114^{\circ}$.

0,2402 Grm. Substanz gaben bei $16^{\circ}$ und $755 \mathrm{Mm}$. Druck 12,8 Ccm. $=$ $6,17 \% \mathrm{~N}$.

$0,1872 \mathrm{Grm}$. Substanz gaben $0,5066 \mathrm{Grm} . \mathrm{CO}_{2}=73,8 \% \mathrm{C}$ und 0,1010 Grm. $\mathrm{H}_{2} \mathrm{O}=5,99 \%$ H.

$\begin{array}{ccc} & \text { Berechnet für } \mathrm{C}_{1 \mathbf{1}} \mathrm{H}_{18} \mathrm{NO}_{2}: & \text { Gefunden: } \\ \mathrm{C} & \mathbf{7 4 , 0 1} & \mathbf{7 3 , 8 \%} \\ \mathrm{H} & 5,72 & \mathbf{5 , 9 9 \%} \\ \mathrm{N} & \mathbf{6 , 1 6} & \mathbf{6 , 1 7} \%\end{array}$

Es liegt also auch in diesem Falle das erwartete Condensationsprodukt, der Cinnamenyl- $\alpha$-cyanacrylsäureester, vor.

\section{Darstellung der Cinnamenyl- $\alpha$-cyanacrylsäure. $\mathrm{C}_{6} \mathrm{H}_{5} . \mathrm{CH}=\mathrm{CH} . \mathrm{CH}=\mathrm{C}$. $(\mathrm{CN})$. $\mathrm{COOH}$.}

$\mathrm{Zu}$ der etwa $60^{\circ}$ heissen alkoholischen Lösung des Esters wurde die berechnete Menge heisser alkoholischer Kalilauge zugefügt, wobei intensive Rothfärbung der Lösung eintrat. Beim Erkalten derselben schied sich ein rothgelb gefärbtes Kaliumsalz ab, das durch Waschen mit absolutem Alkohol von den Verunreinigungen befreit wurde. Dass nur der Ester, nicht auch das Cyan verseift ist, wurde schon durch die Silberbestimmung des aus der wässrigen Kaliumsalzlösung durch salpetersaures Silber dargestellten Silbersalzes bewiesen.

0,1758 Grm. Silbersalz gaben $0,0620 \mathrm{Grm} .=35,27 \% \mathrm{Ag}$.

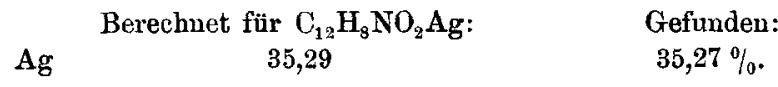

Die freie Säure wurde aus der wässrigen Kaliumsalzlösung durch verdünnte Essigsäure gefällt. Beim Zusatz der letzteren trübt sich die gelbgefärbte Lösung; nach kurzer Zeit, zumal beim Umrühren, ballt sich ein fein vertheilter, Anfangs gelber Niederschlag zusammen, der allmählich sich intensiv roth färbt. Derselbe ist in Alkohol, Aether, Benzol, Chloroform leicht löslich und wird am vortheilhaftesten aus ca. 50procent. Alkohol umkrystallisirt. Dabei löst sich die Säure zuerst wieder klargelb auf und scheidet sich darauf in rothen Flocken wieder $\mathrm{ab}$, die bei $196^{\circ}$ schmelzen. Die Analysen dieser Säure verursachten in Folge der schweren Verbrennbarkeit derselben grosse Schwierigkeiten; deshalb differirten die gefundenen 
Bechert: Ueb. d. Condensat. von Aldehyden u. Cyaniden. 15

Werthe für Kohlenstoff, ausser den unten angeführten, fast immer um $0,5 \%-0,8 \%$ von den berechneten Zahlen.

$0,2430 \mathrm{Grm}$. Substanz gaben bei $16^{\circ}$ und $756 \mathrm{Mm}$. Druck $14,8 \mathrm{Ccm}$. $=$ $7,05 \% \mathrm{~N}$.

$0,1382 \mathrm{Grm}$. Substanz gaben $0,3652 \mathrm{Grm} . \mathrm{CO}_{2}=72,07 \% \mathrm{C}$ und 0,0622 Grm. $\mathrm{H}_{2} \mathrm{O}=4,98 \% \mathrm{H}$.

$\begin{array}{ccc} & \text { Berechnet für } \mathrm{C}_{12} \mathrm{H}_{9} \mathrm{NO}_{2}: & \text { Gefunden: } \\ \mathrm{C} & \mathbf{7 2 , 3 6} & \mathbf{7 2 , 0 7} \% \\ \mathrm{H} & \mathbf{4 , 5 2} & 4,98 \% \\ \mathrm{~N} & \mathbf{7 , 0 3} & \mathbf{7 , 0 5}, \%\end{array}$

Aus dem Kaliumsalz wurde mittelst essigsauren Bleis das gelbe Bleisalz, durch Chlorbaryum das weisse Baryumsalz, durch Eisenchlorid das braune Eisensalz, durch essigsaures Kupfer das grüne Kupfersalz, und durch Chlorcalcium das weisse Calciumsalz dargestellt.

\section{Verhalten des Cinnamenyl- $\alpha$-cyanacrylsäureesters gegen Brom.}

Der in Chloroform gelöste Ester wurde mit zwei Molekülen Brom versetzt, und das Gefäss verschlossen gehalten. In diesem Falle schieden sich keine Krystalle, wie beim pMethoxyphenyl- $\alpha$-cyanacrylsäureester, aus, selbst nach tagelangem Stehen nicht, obgleich das Brom nach und nach verschwand. Erst nachdem das Chloroform an der Luft verdunsten konnte, erstarrte das Ganze zu einem schmutzigweissen Krystallgefüge. Bei dem ganzen Verlauf der Reaction war eine Entwicklung von Bromwasserstoff nicht zu bemerken, welcher Umstand auf eine glatte Addition des Broms schliessen lässt. Aus Alkohol umkrystallisirt, schmilzt der in weissen, glänzenden Blättchen sich abscheidende Bromkörper bei $95^{\circ}$.

0,2256 Grm. Substanz gaben 0,09301 Grm. $=41,23 \%$ Br.

$$
\begin{array}{ccc}
\text { Berechnet für } & \text { Gefunden: } \\
\mathrm{Br} & \mathrm{C}_{8} \mathrm{H}_{5} . \mathrm{CHBr} . \mathrm{CHBr} . \mathrm{CH}=\mathrm{C} .(\mathrm{CN}) \cdot \mathrm{COOC}_{2} \mathrm{H}_{5}: & \\
41,34 & 41,23 \% .
\end{array}
$$

In dem vorliegenden Falle ist also nicht, wie beim vorigen, Bromwasserstoff nach der Addition von Brom abgespalten worden, sondern es ist analog dem Verhalten der Zimmtsäure selbst Brom aufgenommen worden, jedoch nur ein Molekül desselben. Wie durch die obige Formel ausgedrückt werden 
16 Bechert: Ueb. d. Condensat. von Aldehyden u. Cyaniden.

soll, ist die eine Doppelbindung ebenso intact geblieben, wie dies beim $\alpha$-Cyanzimmtsäureester der Fall ist.

Merkwürdiger Weise nimmt die aus dem Ester dargestellte Säure unter denselben Bedingungen kein Brom auf; selbst nach einem Zeitraum von drei Wochen war das Brom noch unverändert in der Cbloroformlösung vorhanden. Beim Verdunsten derselben ging das Brom weg, und das zurückbleibende Gemisch verharzte.

\section{Condensation ron Furfurol und Cyanessigester: \\ Furfur- $u$-cyanacrylsäureester.$$
\left(\mathrm{C}_{4} \mathrm{H}_{3} \mathrm{O}\right) \cdot \mathrm{CH}^{-} \mathrm{C} \cdot(\mathrm{CN}) \cdot \mathrm{COOC}_{2} \mathrm{H}_{5} \text {. }
$$

Bei der Condensation dieser beiden Körper wurde wie oben verfabren. Die Menge des angewandten Natriums, als Aethylat in alkoholischer Lösung, betrug auch hier nur 0,02 Grm. auf etwa $10 \mathrm{Grm}$. des Gemisches. An der Einfallsstelle des Natriumäthylats trat eine intensive violette Färbung auf, die beim Umschütteln der Lösung wieder verschwand. Nach einigen Minuten trat starke Erwärmung und zugleich $\mathbf{A b}$ scheidung von rosaroth gefärbten Nadeln ein. Die Ausbeuten sind fast quantitativ. Die Verbindung ist in Chloroform und Alkohol löslich, und aus letzterem umkrystallisirt, scheidet sie sich in weissen, glänzenden Nadeln von $94^{\circ}$. Schmelzpunkt ab.

0,3232 Grm. Substanz gaben bei $19^{\circ}$ und $749 \mathrm{Mm}$. Druck $21,3 \mathrm{Ccm},=$ $7,47 \% \mathrm{~N}$.

0,1763 Grm. Substauz gaben 0,4048 Grm. $\mathrm{CO}_{2}=62,62 \%$ C und 0,0782 Grm. $\mathrm{H}_{2} \mathrm{O}=4,93 \% \mathrm{H}$.

$\begin{array}{ccc} & \text { Berechnet für } \mathrm{C}_{10} \mathrm{H}_{9} \mathrm{NO}_{3}: & \text { Gefunden : } \\ \mathrm{C} & 62,82 & 62,62 \% \\ \mathrm{H} & 4,71 & 4,93 \% \\ \mathrm{~N} & 7,33 & 7,47 \%\end{array}$

Furfur-a-cyanacrylsäure: $\left(\mathrm{C}_{2} \mathrm{H}_{3} \mathrm{O}\right) \cdot \mathrm{CH}=\mathrm{C}$. $(\mathrm{CN}) \cdot \mathrm{COOH}$.

Der oben beschriebene Ester wurde mit der berechneten Menge alkoholischen Kalis verseift. Zunächst trat intensive Blaufärbung der alkobolischen Lösung ein, aus welcher sich beim Erkalten ein rothbraunes Kaliumsalz abschied. Die Ausbeute ist sehr gering. Das von der bald sich schwarzbraun färbenden Mutterlauge durch Waschen mit absolutem 
Bechert: Ueb. d. Condensat. von Aldehyden u. Cyaniden. 17

Alkohol befreite Kaliumsalz wurde behufs der Darstellung des Silbersalzes in Wasser gelöst und mit salpetersaurem Silber versetzt. Es schied sich dabei ein schmutzigbrauner, flockiger Niederschlag aus, der in Folge seiner raschen Zersetzbarkeit auf seinen Silbergehalt nicht mit Erfolg geprüft werden konnte.

Zur Darstellung der freien Säure wurde das Kaliumsalz mit verdünnter Schwefelsäure zerlegt. Es schied sich ein schwarzgrüner Niederschlag ab, der durch Waschen mit Wasser sich olivengrün färbte. Derselbe ist in heissem Wasser, sowie kaltem Alkohol leicht löslich. Um ihn zu reinigen und umzukrystallisiren, wurde er in ca. 40 procent. alkoholischer Lösung mit Thierkohle mehrere Male ausgekocht und daraus in goldgelben Nadeln von $218^{\circ}$ Schmelzpunkt erhalten.

$0,2208 \mathrm{Grm}$. Substanz gaben bei $15^{\circ}$ und $758 \mathrm{Mm}$. Druck 17,3 Ccm. = $8,76 \% \mathrm{~N}$.

0,1376 Grm. Substanz gaben 0,2970 Grm. $\mathbf{C O}_{2}=58,85 \% \quad \mathrm{C}$ und 0,0414 Grm. $\mathrm{H}_{2} \mathrm{O}=3,34 \% \mathrm{H}$.

$\begin{array}{ccc} & \text { Berechnet für } \mathrm{C}_{8} \mathrm{H}_{5} \mathrm{NO}_{3}: & \text { Gefunden: } \\ \mathrm{C} & 58,9 & 58,85 \% \\ \mathrm{H} & 3,07 & 3, \mathbf{3 4} \% \\ \mathrm{~N} & 8,58 & 8,76 \%\end{array}$

Die Ausbeute an dieser Süure ist in Folge der Zersetzbarkeit des Kaliumsalzes eine sehr geringe, denn aus 10 Grm. Ester wurden nur 0,6 Grm. reine Säure gewonnen.

\section{Verhalten des Furfur- $\alpha$-cyanacrylsäureesters gegen Brom.}

Der Ester wurde in kaltem Chloroform gelöst, und dazu Brom (2 Mol.), gleichfalls in Chloroform gelöst, gefügt. Die Lösung färbte sich dunkelbraun, wobei Entwicklung von Bromwasserstoff eintrat. Da sich aus der Lösung nach einigen Tagen noch nichts ausgeschieden hatte, so wurde das überschüssige Chloroform zum Verdunsten gebracht; die sich alsdann ausscheidende Bromverbindung war sehr verunreinigt durch schmierige Beimengungen, die aber durch öfteres Behandeln der ersteren mit Thierkohle sich entfernen liessen. Der Schmelzpunkt der so erhaltenen Verbindung lag zwischen $80^{\circ}$ und $83^{\circ}$. Zwei Brombestimmungen ergaben $19,7 \%$, resp. $19,5 \%$, während bei der erwarteten Verbindung 29,63\% Brom 
18 Bechert: Ueb. d. Condensat. von Aldehyden u. Cyaniden.

hätten gefunden werden müssen. Aehnliche ungünstige Ergebnisse traten ein, wenn man die Lösung länger stehen liess.

Mit Berücksichtigung dieser Erfahrung, dass in Chloroform die Umsetzung so schwer von statten ging, wurde nun als Lösungsmittel heisser Eisessig zur Anwendung gebracht. In der eben genügenden Menge desselben aufgelöst, wurde der Ester mit dem gleichfalls in Eisessig gelösten Brom zusammengebracht, wobei starke Bräunung der Lösung eintrat. Nach einigen Stunden hatte sich ein Niederschlag abgeschieden, der aus feinen, durch eine schmierige Beimengung sehr verunreinigten Nadeln bestand. Aus der Mutterlauge schied sich beim Zusatz von Wasser eine grosse Menge feiner Nadeln ab, die aus Alkohol umkrystallisirt wurden, wobei sie sich als goldgelbe Nadeln von $111^{\circ}$ Schmelzpunkt absetzten. Der direct entstandene Niederschlag wurde durch Auswaschen mit Eisessig von der schmierigen Beimengung befreit und dann gleichfalls aus Alkohol umkrystallisirt. Er erwies sich als identisch mit dem durch Wasser ausgefällten Produkte.

$0,2829 \mathrm{Grm}$. Substanz gaben 0,08449 Grm. $=29,86 \% \mathrm{Br}$.

$0,3153 \mathrm{Grm}$. Substanz gaben $0,09424 \mathrm{Grm} .=29,88 \% \mathrm{Br}$.

Berechnet für $\left(\mathrm{C}_{4} \mathrm{H}_{3} \mathrm{O}\right) \mathrm{CBr}=\mathrm{C}$. (CN). $\mathrm{COOC}_{2} \mathrm{H}_{5}$ : Gefunden:

$$
\begin{array}{llll}
\mathrm{Br} & 29,63 & 29,86 & 29,88 \%
\end{array}
$$

Der Erfolg der Einwirkung von Brom auf den Furfur- $\alpha-$ cyanacrylsäureester ist also ein gleicher wie beim p-Methoxyphenyl- $\alpha$-cyanacrylsäureester.

Condensation von Piperonal und Cyanessigester:

Methylendioxyphenyl- $\alpha$-cyanacrylsäureester.

$$
\mathrm{C}_{8} \mathrm{H}_{3}\left(\mathrm{O}_{2} \mathrm{CH}_{2}\right) \mathrm{CH}=\mathrm{C} \text {. }(\mathrm{CN}) . \mathrm{COOC}_{2} \mathrm{H}_{5} \text {. }
$$

2,5 Grm. Piperonal wurden mit 2 Grm. Cyanessigester in $10 \mathrm{Grm}$. absolutem Alkohol gelöst und dazu 0,04 Grm. Natrium als Aethylat hinzugefügt. Unter anfänglich geringer Erwärmung trat nach wenigen Minuten eine Abscheidung von gelben Krystallblättchen ein. Dieselben sind in Alkohol, Benzol und Chloroform löslich; aus erstgenanntem Lösungsmittel umkrystallisirt, schmelzen sie bei $106^{\circ}$. $5,87 \% \mathrm{~N}$.

$0,2590 \mathrm{Grm}$. Substanz gaben bei $17^{\circ}$ und $755 \mathrm{Mm}$. Druck $13,2 \mathrm{Ccm} .=$

0,1119 Grm. Substanz gaben 0,2600 Grm. $\mathrm{CO}_{2}=63,45 \% \mathrm{C}$ und 0,0478 Grm. $\mathrm{H}_{2} \mathrm{O}=4,73 \% \mathrm{H}$. 
Bechert: Ueb. d. Condensat. von Aldehyden u. Cyaniden. 19

$\begin{array}{ccc} & \text { Berechnet für } \mathrm{C}_{13} \mathrm{H}_{11} \mathrm{NO}_{4}: & \text { Gefurden: } \\ \mathrm{C} & 63,67 & 63,45 \% \\ \mathrm{H} & 4,5 & 4,73 \% \\ \mathrm{~N} & 5,71 & 5,88 \%\end{array}$

Methylendioxy phenyl- $\alpha$-cyanacrylsäure. $\mathrm{C}_{6} \mathrm{H}_{3}\left(\mathrm{O}_{2} \mathrm{CH}_{2}\right) \mathrm{CH}=\mathrm{C}$. $(\mathrm{CN})$. $\mathrm{COOH}$.

Bei der Darstellung dieser Säure wurde wie bei der Gewinnung der vorher beschriebenen Säuren verfahren, indem das Kaliumsalz, das sich beim Verseifen des Esters aus der heissen alkoholischen Lösung als weisser Niederschlag absetzte, durch verdünnte Essigsäure zerlegt wurde. Die $\mathrm{Zu}$ sammensetzung des aus dem Kaliumsalze dargestellten Silbersalzes zeigte, dass auch hier die Cyangruppe nicht angegriffen worden ist.

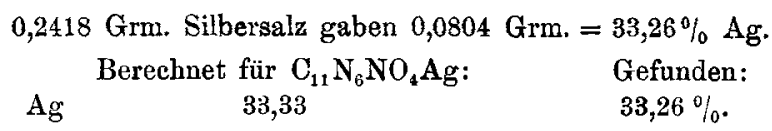

Die Methylendioxyphenyl- $\alpha$-cyanacrylsäure, die in Alkohol, Benzol und Aether löslich ist, scheidet sich aus ersterem Lösungsmittel in gelben Nadeln ron $230^{\circ}$ Schmelzpunkt ab. $6,55 \% \mathrm{~N}$.

$0,3528 \mathrm{Grm}$. Substanz gaben bei $12^{\circ}$ und $758 \mathrm{Mm}$. Druck 19,6 Ccm. $=$

0,1564 Grm. Substanz gaben 0,3470 Grm. $\mathbf{C O}_{2}=60,59 \%$ C und 0,0466 Grm. $\mathrm{H}_{2} \mathrm{O}=3,31 \% \mathrm{H}$.

$\begin{array}{ccc} & \text { Berechnet für } \mathrm{C}_{11} \mathrm{H}_{7} \mathrm{NO}_{4}: & \text { Gefunden: } \\ \mathrm{C} & 60,83 & 60,59 \% \\ \mathrm{H} & 3,22 & 3,31 \% \\ \mathrm{~N} & 6,43 & 6,55 \%\end{array}$

Verhalten des Methylendioxyphenyl-a-cyanacrylsäureesters gegen Brom.

Bei der Behandlung dieses Esters in Chloroformlösung mit Brom wurden dieselben negativen Resultate beobachtet wie beim Furfur- $\alpha$-cyanacrylsäureester, und deshalb wurde, wie bei jenem, Eisessig als Lösungsmittel angewandt. Die entstandene Bromverbindung schied sich aus dem Eisessig in gut ausgebildeten gelben Krystallen aus, die bei $131^{\circ}$ schmolzen.

$0,3660 \mathrm{Grm}$. Substanz gaben 0,08979 Grm. $=24,51 \% \mathrm{Br}$.

$0,4232 \mathrm{Grm}$. Substanz gaben $0,10349 \mathrm{Grm} .=24,45 \% \mathrm{Br}$. 
20 Bechert: Ueb. d. Condensat. von Aldehyden u. Cyaniden. Br

Berechnet für $\mathrm{C}_{6} \mathrm{H}_{3}\left(\mathrm{O}_{2} \mathrm{CH}_{2}\right) \mathrm{CBr}=\mathrm{C}$.(CN).COOC $\mathrm{H}_{5}$ : Gefunden: 24,69 $24,5124,45 \%$.

Mit der Darstellung dieser Bromverbindung schliesst die Reihe der Condensationsprodukte ab, die sich, an die Arbeit von Carrick anschliessend, als Abkömmlinge der $\alpha$-Cyanacrylsäure betrachten liessen.

Ein grosser Unterschied tritt zwischen den obigen Estern und dem $\alpha$-Cyanzimmtsäureester hervor, und zwar in ihrem Verhalten gegen Brom; während letzterer gar nicht angegriffen wird, wie durch mehrfache von mir angestellte Versuche bestätigt wurde, reagirt Brom mit den oben beschriebenen Estern leicht, wenn auch nicht in gleicher Weise.

Die folgenden Versuche zeigen, dass Salicylaldehyd sich mit Cyanessigester in anderer Weise condensirt, als die vorher angewandten Aldehyde, und dass das Produkt der Reaction sich ganz eigenthümlich verhält.

Condensation von Salicylaldehyd und Cyanessigester: o-Oxybenzaldicyanessigester.

$$
\mathrm{C}_{6} \mathrm{H}_{4} \cdot(\mathrm{OH}) \cdot \mathrm{CH}\left\langle\begin{array}{l}
\mathrm{CH} \cdot(\mathrm{CN}) \cdot \mathrm{COOC}_{2} \mathrm{H}_{5} \\
\mathrm{CH} \cdot(\mathrm{CN}) \cdot \mathrm{COOC}_{2} \mathrm{H}_{5}
\end{array}+1 / 2 \mathrm{H}_{2} \mathrm{O}\right. \text {. }
$$

Molekulare Mengen von Salicylaldehyd und Cyanessigester wurden, in wenig Alkohol gelöst, mit geringen Quantitäten Natriumäthylat versetzt. Die erst schwachgelb gefärbte Lösung nahm eine intensiv weinrothe Färbung an, die, je nach der angewandten Menge Natriumäthylats, sich bald dunkelroth bis braun färbte. Nach einiger Zeit erfolgte intensive Wärmeentwicklung und zugleich Abscheidung von Krystallen. Sie wurden mit Alkohol gewaschen und aus demselben Lösungsmittel umkrystallisirt. Der Schmelzpunkt des neutral reagirenden, in weissen, hartkörnigen Krystallen sich abscheidenden Körpers liegt bei $140^{\circ}$. Ausser in Alkohol ist er noch in Chloroform, Benzol, Aether und Eisessig löslich.

Auch in dem vorliegenden Falle war ein einfaches Condensationsprodukt erwartet worden; aber da in den angestellten Analysen der Kohlenstoffgehalt um etwa $6 \%$ von dem auf ein solches Produkt berechneten differirte, und da bei der Bildung des Körpers stets unverbrauchter Salicylaldehyd in der Mutter- 
Bechert: Ueb. d. Condensat. von Aldehyden u. Cyaniden. 21

lauge sich vorfand, so lag die Vermuthung nahe, dass die Reaction verschieden von den oben beschriebenen verlaufen war. Um den überschüssigen Salicylaldehyd zur Wirkung zu bringen, wurden nun zwei Moleküle Cyanessigester auf ein Molekül Salicylaldehyd angewandt, und dabei die Wahrnehmung gemacht, dass diese Menge Salicylaldehyd vollständig in Reaction getreten war. Auch die Thatsache, dass früher bei Anwendung von einem Molekül Cyanessigester die Ausbeuten nur etwa $60 \%$ der berechneten betrugen, jetzt aber fast quantitativ ausfielen, berechtigte $\mathrm{zu}$ der Annahme, dass sich ein Molekül Salicylaldehyd mit zwei Molekülen Cyanessigester unter Wasseraustritt condensirt hatten. Doch auch für diese Verbindung konnten keine stimmenden Analysen erhalten werden. Wegen des geringen Kohlenstoffgehaltes lag die Vermuthung nahe, dass der Ester wasserhaltig sei; die in Folge dessen angestellten Wasserbestimmungen bestätigten die Annahme; es ergab sich, dass der Ester ein halbes Molekül Wasser, also auf zwei Moleküle Ester ein solches, enthält. Obgleich nun dieses Verhältniss etwas befremdend war, so muss doch bemerkt werden, dass gerade Salicylaldehydverbindungen öfters mit einhalb Molekül, ja sogar mit dreiviertel Molekül Wasser krystallisiren.

0,4644 Grm. Substanz verloren beim Erhitzen auf $105^{\circ} 0,0120 \mathrm{Grm}$. $\mathrm{H}_{2} \mathrm{O}=2,59 \% \quad \mathrm{H}_{2} \mathrm{O}$.

0,4786 Grm. Substanz verloren unter denselben Bedingungen $0,0132 \mathrm{Grm}_{*}=2,74 \% \mathrm{H}_{2} \mathrm{O}$.

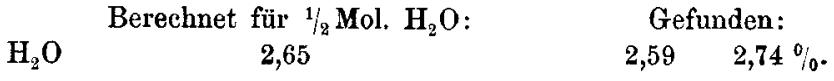

$0,1666 \mathrm{Grm}$. des wasserhaltigen Esters gaben 0,3672 Grm. $\mathrm{CO}_{2}=$ $60,12 \% \mathrm{C}$ und 0,0856 Grm. $\mathrm{H}_{2} \mathrm{O}=5,70 \% \mathrm{H}$.

0,1554 Grm. Substanz gaben 0,3414 Grm. $\mathrm{CO}_{2}=59,92 \% \mathrm{C}$ und 0,0852 Grm. $\mathrm{H}_{2} \mathrm{O}=6,01 \% \mathrm{H}$.

0,2470 Grm. Substanz gaben 0,5436 Grm. $\mathrm{CO}_{2}=60,02 \% \mathrm{C}$ und 0,1230 Grm. $\mathrm{H}_{2} \mathrm{O}=5,54 \stackrel{\circ}{\circ} \mathrm{H}$.

0,1548 Grm. Substanz gaben 0,3424 Grin. $\mathrm{CO}_{2}=60,45 \% \mathrm{C}$ und 0,0804 Grm. $\mathrm{H}_{2} \mathrm{O}=5,77 \% \mathrm{H}$.

$0,5150 \mathrm{Grm}$. Substanz gaben hei $17^{\circ}$ und $755 \mathrm{Mm}$. Druck $37,4 \mathrm{Ccm}$. = $8,37 \% \mathrm{~N}$.

$0,5882 \mathrm{Grm}$. Substanz gaben bei $12^{\circ}$ und $760 \mathrm{Mm}$. Druck $42,8 \mathrm{Ccm} .=$ $8,6 \% \mathrm{~N}$. 
22 Bechert: Ueb. d.Condensat. von Aldehyden u. Cyaniden.

\begin{tabular}{cccccc}
\multicolumn{3}{c}{ Berechnet für } & \multicolumn{5}{c}{ Gefunden: } \\
$\mathrm{C}$ & $\mathrm{C}_{17} \mathrm{H}_{18} \mathrm{~N}_{2} \mathrm{O}_{5}+1 / 2 \mathrm{H}_{2} \mathrm{O}:$ & & & & \\
$\mathrm{H}$ & 60,18 & 60,12 & 59,92 & 60,02 & $60,45 \%$ \\
$\mathrm{~N}$ & 5,60 & 5,70 & 6,01 & 5,54 & $5,77 \%$
\end{tabular}

Durch diese Reihe von Analysen dürfte die Zusammensetzung dieses Esters wohl erwiesen sein.

Als Argument für die Richtigkeit der Constitution des o-Oxybenzaldicyanessigesters möge Folgendes angeführt sein:

Ein Körper, der die beiden Carboxäthylgruppen in solcher Function wie der obige enthält, sollte mit alkoholischem Ammoniak ein Imid liefern. Zur Prüfung dieser Vermuthung wurde der Ester in absolutem Alkohol gelöst und mit alkoholischem Ammoniak versetzt. In der Kälte trat keine Reaction ein. Nachdem jedoch die Lösung ein Mal aufgekocht worden war, schied sich ein blättrig-krystallinischer Niederschlag aus der heissen Lösung ab. Derselbe ist in Aether, Benzol, Chloroform, Petroläther und Wasser unlöslich, in Alkohol nur spurenweise, in Eisessig dagegen äusserst leicht löslich. Daher wurden beim Umkrystallisiren zu dem absoluten Alkohol einige Tropfen Eisessig zugesetzt; das Imid schied sich in grauweissen Flocken aus; es schmolz bei $280^{\circ}$ noch nicht.

$0,1500 \mathrm{Grm}$. Substanz gaben bei $20^{\circ}$ und $760 \mathrm{Mm}$. Druck $21,8 \mathrm{Ccm} .=$ $16,63 \% \mathrm{~N}$.

$$
\begin{aligned}
& \text { Berechnet für } \\
& \text { Gefunden: } \\
& \mathrm{C}_{6} \mathrm{H}_{4} \cdot(\mathrm{OH}), \mathrm{CH}\left\langle\begin{array}{l}
\mathrm{CH} .(\mathrm{CN}) . \mathrm{CO} \\
>\mathrm{NH}:
\end{array}\right. \\
& 16,63 \% \text {. }
\end{aligned}
$$

Auch die folgenden Versuche haben Ergebnisse geliefert, die mit obiger Annahme in Einklang stehen.

Kalium- und Silbersalz der o-Oxybenzaldicyanessigsäure. - Um die obigem Ester entsprechende Säure zu erhalten, wurde, analog der Darstellung der früher erhaltenen Säuren, zunächst das Kaliumsalz dargestellt. Aus der heissen alkoholischen Lösung, die sich beim Versetzen mit der berechneten Menge heissen alkoholischen Kalis intensiv roth färbte, schied sich das Kaliumsalz als gelbes Krystallpulver aus. Stehenlassen an einem warmen Orte begünstigt die Ausbeute. Das Kaliumsalz liefert mit salpetersaurem Silber ein gelbes Silbersalz. 
Bechert: Ueb. d.Condensat. von Aldehyden u. Cyauiden. 23

0,0430 Grm. Silbersalz gaben $0,0192 \mathrm{Grm} .=44,66 \% \mathrm{Ag}$.

0,1606 Grm. Silbersalz gaben 0,0718 Grm. $=44,70 \% \mathrm{Ag}$.

0,2912 Grm. Silbersalz gaben $0,1290 \mathrm{Grm},=44,29 \% \mathrm{Ag}$.

Berechnet für

Gefunden:

$$
\begin{array}{llll}
\mathrm{C}_{6} \mathrm{H}_{4} \cdot(\mathrm{OH}) \cdot \mathrm{CH} / \mathrm{CH} \cdot(\mathrm{CN}) \cdot \mathrm{COOAg} \\
\mathrm{Ag} \cdot(\mathrm{CH}) \cdot \mathrm{COOAg}
\end{array}
$$

Nur eine Carboxäthylgruppe zu verseifen, gelang nicht, vielmehr wurde bei diesen Versuchen nur die Ausbeute beeinträchtigt.

Da bei der Zerlegung des Kaliumsalzes durch verdünnte Säure nicht die erwartete Säure zu gewinnen war, so wurde auch die Verbrennung des Silbersalzes vorgenommen, um zu sehen, ob es wirklich, wie oben angegeben, zusammengesetzt sei.

$0,1366 \mathrm{Grm}$. Silbersalz gaben $0,1576 \mathrm{Grm} . \mathrm{CO}_{2}=31,46 \% \mathrm{C}$ und 0,0148 Grm. $\mathrm{H}_{2} \mathrm{O}=1,2 \% \mathrm{H}$

0,2594 Grm. Silbersalz gaben 0,3015 Grm. $\mathrm{CO}_{2}=31,73 \% \mathrm{C}$ und 0,0406 Grm. $\mathrm{H}_{2} \mathrm{O}=1,77 \% \mathrm{H}$.

\begin{tabular}{cccc} 
& Berechnet für $\mathrm{C}_{13} \mathrm{H}_{8} \mathrm{~N}_{2} \mathrm{O}_{5} \mathrm{Ag}_{2}:$ & \multicolumn{2}{c}{ Gefunden: } \\
$\mathrm{C}$ & 31,96 & 31,46 & $31,73 \%$ \\
$\mathrm{H}$ & 1,63 & 1,2 & $1,77 \%$
\end{tabular}

Zersetzugsprodukt des Kaliumsalzes durch verdünnte Säure:

Cyancumarin.<smiles>CCCCCCCOCC(C#N)C#N</smiles>

Versetzt man die wässrige Lösung des Kaliumsalzes mit verdünnter Schwefelsäure, so entfärbt sich die gelbe Lösung, und es scheidet sich ein in Nadeln krystallisirter grauweisser Niederschlag aus. Dieser neutral reagirende Körper, der sich am besten aus absolutem Alkohol umkrystallisiren lässt, schmilzt bei $182^{\circ}$. Er ist löslich in Benzol und lässt sich aus der Benzollösung durch Petroläther ausfällen. Das so erhaltene leichte, flockige, amorphe Produkt wird beim Reiben stark elektrisch. Wird die alkoholische Lösung des Körpers mit Eisenchlorid versetzt, so tritt keine Violettfärbung ein, was darauf schliessen lässt, dass das Hydroxyl des Salicylaldehyds nicht mebr in der Verbindung enthalten ist. 
24 Bechert: Ueb. d.Condensat. von Aldehyden u. Cyaniden. $8,45 \% \mathrm{~N}$.

$0,2378 \mathrm{Grm}$. Substanz gaben bei $14^{\circ}$ und $764 \mathrm{Mm}$. Druck $17 \mathrm{Ccm}$. =

$0,1440 \mathrm{Grm}$. Substanz gaben bei $13^{\circ}$ und $758 \mathrm{Mm}$. Druck $10,2 \mathrm{Ccm} .=$ $8,34 \% \mathrm{~N}$. $8,2 \% \mathrm{~N}$.

$0,2203 \mathrm{Grm}$. Substanz gaben bei $21^{\circ}$ und $753 \mathrm{Mm}$. Druck $16,0 \mathrm{Ccm} .=$

$0,1666 \mathrm{Grm}$. Substanz gaben $0,4306 \mathrm{Grm} . \mathrm{CO}_{2}=70,46 \% \mathrm{C}$ und 0,0468 Grm. $\mathrm{H}_{2} \mathrm{O}=3,12 \% \mathrm{H}$.

$0,1478 \mathrm{Grm}$. Substanz gaben 0,3806 Grm. $\mathrm{CO}_{2}=70,23 \% \mathrm{C}$ und 0,0418 Grm. $\mathrm{H}_{2} \mathrm{O}=3,19 \% \mathrm{H}$. überein.

Die gefundenen Werthe stimmen mit denen der Formel $\mathrm{C}_{10} \mathrm{H}_{3} \mathrm{NO}_{2}$

$\begin{array}{cc} & \text { Berechnet: } \\ \text { C } & \mathbf{7 0 , 1 8} \\ \text { H } & \mathbf{2 , 9 2} \\ \text { N } & 8,18\end{array}$

\begin{tabular}{ccc}
\multicolumn{5}{c}{ Gefunden: } \\
- & $\mathbf{7 0 , 4 6}$ & $\mathbf{7 0 , 2 3} \%$ \\
- & 3,12 & $3,19 \%$ \\
8,2 & 8,34 & $8,45 \%$
\end{tabular}

Die aus dem Kaliumsalz erwartete Säure hätte 56,93\% $\mathrm{C}$ und $3,65 \%$ $H$ enthalten müssen.

Um die Constitution dieser Verbindung zu ergründen, wurde zunächst versucht, ihr Silbersalz darzustellen. Doch gelang dies auf keine Weise. Beim Veraschen des Körpers auf dem Platinblech machte sich ein intensiver Cumaringeruch geltend, und dieser zuerst unbeachtete Umstand leitete auf den richtigen Weg zur Erkenntniss der Constitution obiger Verbindung. Die empirisch für dieselbe abgeleitete Formel unterscheidet sich von der des Cumarins nur dadurch, dass in letzterer ein Wasserstoffatom durch die Cyangruppe substituirt anzunehmen ist. Die Entstehung dieses Cyancumarins aus dem o-Oxybenzaldicyanessigester wird leicht verständlich, wenn man annimmt, dass aus der dem Ester entsprechenden, für sich nicht beständigen Säure Cyanessigester und Wasser abgespalten wird.

Wenn nun das Cyancumarin vorlag, so musste dasselbe, zweckmässig mit Kalilauge behandelt, das Kaliumsalz der Cumarincarbonsäure ${ }^{1}$ ) liefern. $\mathrm{Zu}$ diesem $Z_{\text {wecke wurde das }}$ Cyancumarin mit wässrigem Kalihydrat gekocht, und zwar nur 5-10 Minuten lang, da sonst weitergehende Zersetzung erfolgt. Beim Abkühlen scheidet sich dann ein gelbgefärbtes Kaliumsalz $a b$, das in wässriger Lösung durch verdünnte

1) Ber. 19, Ref. 350 . 
Schwefelsäure zerlegt wird, nnd dabei einen weissen, krystallinischen Niederschlag giebt, der aus absolutem Alkohol umkrystallisirt bei $187^{\circ}$ schmilat. Dieser so entstandene Körper ist den Reactionen und dem Aussehen nach zweifellos Cumarincarbonsäure (siehe unten).

Directe Darstellung der Cumarincarbonsäure aus dem o-Oxybenzaldicyanessigester.<smiles>O=C(O)C(=O)OC[18OH]</smiles>

Eine einfache Ueberlegung musste zu dem Ergebniss führen, dass der 0-Oxybenzaldicyanessigester mit einem genügenden Ueberschuss von Alkali behandelt das Salz der Cumarincarbonsäure direct liefern sollte. Zu diesem $\mathrm{Z}_{\text {wecke }}$ wurde der Ester mit etwas mehr als der berechneten Menge, z. B. der anderthalbfachen, wässriger Kalilauge verseift, wobei starke Ammoniakentwicklung auftrat. Je nach der Länge der Einwirkung von dem Alkali und der angewandten Menge desselben schieden sich geringere oder grössere Mengen eines Kaliumsalzes ab, das sich aber noch als saures Salz erwies. Aus der Mutterlauge desselben wurde durch verdünnte Schwefelsäure ein sauer reagirendes Produkt ausgeschieden, das mit dem Körper, der durch Zerlegung des erwähnten Kaliumsalzes mittelst verdünnter Säure erhalten wurde, identisch war. Da diese Produkte beim Veraschen auf Platin noch Alkali hinterliessen, so wurden sie durch Kochen mit halbverdünnter Salzsäure vollends verseift, und es schieden sich beim Erkalten der Salzsäurelösung weisse Nadeln aus, die sich alkalifrei erwiesen. Dieselben sind in Aether, Benzol, Petroläther unlöslich, werden durch Kochen mit Wasser nicht verändert und lassen sich aus heissem Alkohol umkrystallisiren, wobei sie sich in weissen Nadeln von $187^{\circ}-188^{\circ}$ Schmelzpunkt absetzen. Der Körper reagirt in absolutem Alkohol schwach sauer, in verdünntem aber stark sauer. Die durch die Elementaranalyse gefundenen Zahlenwerthe stimmen mit denen der Cumarincarbonsäure überein.

$0,1936 \mathrm{Grm}$. Substanz gaben $0,4468 \mathrm{Grm} . \mathrm{CO}_{2}=62,94 \% \mathrm{C}$ und 0,0594 Grm. $\mathrm{H}_{2} \mathrm{O}=3,41 \% \mathrm{H}$.

$0,1732 \mathrm{Grm}$. Substanz gaben $0,3994 \mathrm{Grm} . \mathrm{CO}_{2}=62,87 \% \mathrm{C}$ und $0,0524 \mathrm{Grm} . \mathrm{H}_{2} \mathrm{O}=3,36 \% \mathrm{H}$. 
26 Bechert: Ueb. d. Condensat. von Aldehyden u. Cyaniden.

\begin{tabular}{cccc} 
& Berechnet für $\mathrm{C}_{10} \mathrm{H}_{6} \mathrm{O}_{4}:$ & \multicolumn{2}{c}{ Gefunden; } \\
$\mathrm{C}$ & 63,15 & 62,94 & $62,87 \%$ \\
$\mathrm{H}$ & 3,15 & 3,41 & $3,36 \%$
\end{tabular}

Bei der Bildung dieser Säure ist also ein Molekül Cyanessigester, resp. Kaliumsalz abgespalten und zu Malonsäure verseift worden; das vorübergehend entstehende Salz der o-Oxybenzalcyanessigsäure ist sodann in o-Oxybenzalmalonsäure übergeführt worden, welche, in Freiheit gesetzt, sich unter Abspaltung ron Wasser in Cumarincarbonsäure umsetzt:

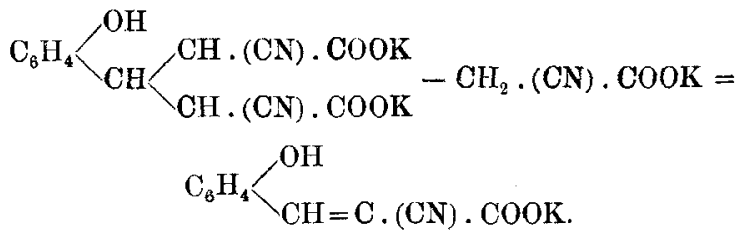

Daraus entsteht durch weiteres Verseifen und Behandeln mit Salzsäure:<smiles>CCCC=CC(=O)C(=O)O</smiles>

das durch Wasserabspaltungen in

übergeht.<smiles>O=C(O)C(=Cc1ccccc1)C(=O)O</smiles>

Die abgespaltene Malonsäure konnte sowohl durch ihren Schmelzpunkt, als auch durch ihre Metallsalze nachgewiesen werden.

Von der Cumarincarbonsäure wurde aus ihrer durch Ammoniak neutralisirten Lösung das Silbersalz dargestellt.

$0,2184 \mathrm{Grm}$. Silbersalz gaben $0,0800 \mathrm{Grm} . \mathrm{Ag}=36,6 \%$

$\mathrm{Ag}$

Berechnet für $\mathrm{C}_{10} \mathrm{H}_{5} \mathrm{O}_{4} \mathrm{Ag}$ :

36,36

Gefunden: $36,6 \%$.

Mittelst Chlorbaryum wird ein Baryumsalz gefällt. 0,4157 Grm. Baryumsalz gaben 0,1885 Grm. $\mathrm{BaSO}_{4}=26,66 \% \mathrm{Ba}$.

Berechnet für

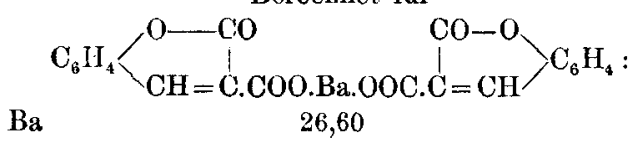

Gefunden:

$26,66 \%$. 
Bechert: Ueb. d. Condensat. von Aldehyden u. Cyaniden. 27

Das Amid der Cumarincarbonsäure, aus letzterer durch Kochen mit alkoholischem Ammoniak erhalten, ist in den bekannten Lösungsmitteln unlöslich und kann nur aus Alkohol, dem Eisessig beigemischt ist, umkrystallisirt werden. Die dabei erhaltenen grauweissen, flockigen Massen schmelzen bei $236^{\circ}$.

Die Stickstoffbestimmung von dem nur mit Alkohol gewaschenen direct gewonnenen Amid ergab 7,03\%, während 7,40\% berechnet sind.

Verhalten des o-Oxybenzaldicyanessigesters gegen Brom.

Der in Chloroform gelöste Ester wurde mit Brom (Ueberschuss schadet nicht) versetzt. Nach kurzer Zeit schied sich ohne Bromwasserstoffentwicklung ein orangerothes Krystallpulver aus, das auf dem Filter mit Chloroform ausgewaschen wurde. In Alkohol; heissem Chloroform ist der erhaltene Bromkörper sehr leicht löslich, desgleichen in Aether und Benzol. Am besten lässt er sich aus Eisessig umkrystallisiren. Durch Kochen mit Wasser wird er zerstört. Der Schmelzpunkt des neutral reagirenden Körpers liegt zwischen $125^{\circ}$ und $128^{\circ}$.

Von zahlreichen Analysen, die mit verschiedenen Präparaten vorgenommen wurden, seien nur die Endergebnisse mitgetheilt.

Am besten stimmen die gefundenen Werthe auf die empirische Formel $\mathrm{C}_{11} \mathrm{H}_{8} \mathrm{NO}_{3} \mathrm{Br}_{3}$.

Für dieselbe wurden

\begin{tabular}{lcrrr} 
& berechnet: & \multicolumn{3}{c}{ Gefunden: } \\
$\mathrm{C}$ & 29,86 & - & 29,73 & $28,88 \%$ \\
$\mathrm{H}$ & 1,55 & - & 2,47 & $2,70 \%$ \\
$\mathrm{~N}$ & 3,16 & 2,96 & 2,90 & $2,87 \%$ \\
$\mathrm{Br}$ & 54,3 & 54,8 & 54,47 & $54,91, \ldots$
\end{tabular}

Eine Constitutionsformel $\mathrm{zu}$ finden, ist trotz aller angestellten Versuche, den Körper dureh Reduction in eín bromfreies Produkt zu verwandeln, bis jetzt noch nicht möglich gewesen.

Wie aus den vorliegenden Resultaten zu ersehen, verlaufen die Condensationen von Aethylencyanid und Aldehyden je nach den Bedingungen verschieden, in keinem Falle sind Produkte von der erwarteten Zusammensetzung

$$
\begin{array}{ll}
\mathrm{CR}^{\prime \prime} . \mathrm{CN} & \mathrm{CR}^{\prime \prime} . \mathrm{CN} \\
\mathrm{CH}_{2}, \mathrm{CN} & \text { oder } \quad \mathrm{CR}^{\prime \prime}, \mathrm{CN}
\end{array}
$$


28 Bechert: Ueb. d. Condensat. von Aldehyden u.Cyaniden.

entstanden, sondern es hat sich gezeigt, dass stets Aufnahme von Wasser neben der Condensation mit dem betreffenden Aldehyd stattfindet.

Cyanessigester dagegen condensirt sich in den meisten Fällen leicht mit den angewandten Aldehyden so, wie erwartet war; nur bei Anwendung von Salicylaldehyd machen sich andere Verhältnisse geltend, da erstens 2 Mol. Cyanessigester, statt $1 \mathrm{Mol}$, sich mit $1 \mathrm{Mol}$. Salicylaldehyd vereinigen, und zweitens bei der Weiterverarbeitung des erhaltenen $\mathbf{p}$-MethoxyphenyldiCyanessigesters weitere intramolekulare Veränderungen erfolgen.

Zum Schluss möge noch das verschiedene Verhalten der gleichartig zusammengesetzten Condensationsprodukte aus Cyanessigester mit den verschiedenen Aldehyden gegen Brom hervorgehoben werden. Bei dem einfachsten Vertreter dieser Körperreihe, dem $\alpha$-Cyanzimmtsäureester,

$$
\mathrm{O}_{6} \mathrm{H}_{5} . \mathrm{CH}=\mathrm{O} . \mathrm{CN} . \mathrm{COO} . \mathrm{C}_{6} \mathrm{H}_{5} \text {, }
$$

tritt Brom merkwürdiger Weise nicht in die Verbindung ein, wie von Carrick gezeigt, und auch von mir durch eine Reihe diesbezüglicher Versuche bestätigt worden ist. Ist jedoch das Phenyl jenes Esters durch p-Methoxyphenyl ersetzt, su tritt Brom glatt in Reaction, indem es zuerst unter Lösung der doppelten Bindung ein Additionsprodukt bildet, das aber unter Bromwasserstoffabspaltung und Wiederherstellung der doppelten Bindung in ein Substitutionsprodukt übergeht. Ebenso verhält es sich, wenn statt des Phenyls das Radikal des Furfurols $\mathrm{C}_{4} \mathrm{H}_{3} \mathrm{O}$ und das des Piperonals $\mathrm{C}_{6} \mathrm{H}_{3} \mathrm{O}_{2} \mathrm{CH}_{2}$ in die Verbindung eingeführt sind. Nur in einem Falle, bei dem Cinnamenyl- $\omega$-cyanacrylsäureester, verhält es sich anders. Derselbe addirt, gleich dem Zimmtsäureester, $1 \mathrm{Mol}$. Brom. Die zweite in ihm anzunehmende doppelte Bindung bleibt von der Wirkung des Broms ebenso unberührt, wie die des $\alpha$-Cyanzimmtsäureesters.

Das Bromprodukt des o-Oxybenzaldicyanessigesters dürtte, wie die Analysen ergeben haben, ein Spaltungsprdukt sein, dessen Constitution noch nicht aufgeklärt worden ist.

Leipzig, im October 1893. 